Supporting Information

For

\title{
Comparison of contributions to chloride in urban stormwater from winter brine and rock salt application
}

\author{
By
}

Danelle M. Haake and Jason H. Knouft

Saint Louis University

Pages S1 - S10

Including: Figures S1-S3

Tables S1-S5 
Figure S1. Linear regression between paired specific conductance and chloride measurements from stormwater grab samples in St. Louis County, Missouri. For conductance $<2.44 \mathrm{mS} / \mathrm{cm}$ [chloride $=195 *$ conductance -77.4 ], adjusted R-squared $=0.792$ (light gray line); for conductance $\geq 2.44 \mathrm{mS} / \mathrm{cm}$ [chloride $=382 *$ conductance -427 ], adjusted R-squared $=0.996$ (dark gray line).

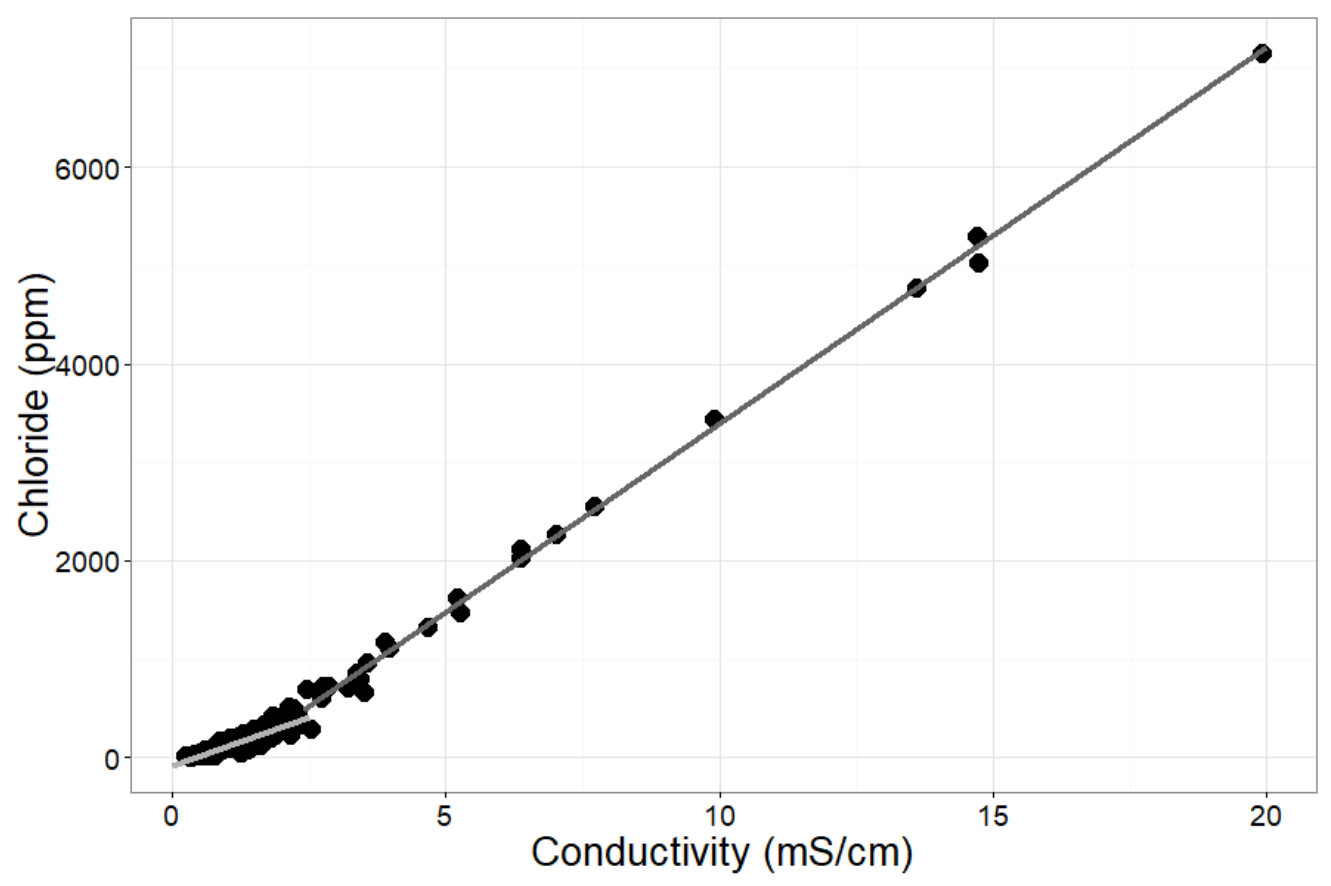


Figure S2. Linear regression of chloride loads for each storm in paired cities, including $95 \%$ confidence interval. This is the same data as Figure 3 except that the storm event with the greatest overall chloride load was removed to ensure that the removed data point was not driving the relationship. Each point represents the chloride load from a pair of cities during an individual storm event (triangles: Ferguson/Jennings; squares: Manchester/Ballwin; circles: Rock Hill/Webster Groves). The dashed 1:1 line represents equal salt application in paired cities.

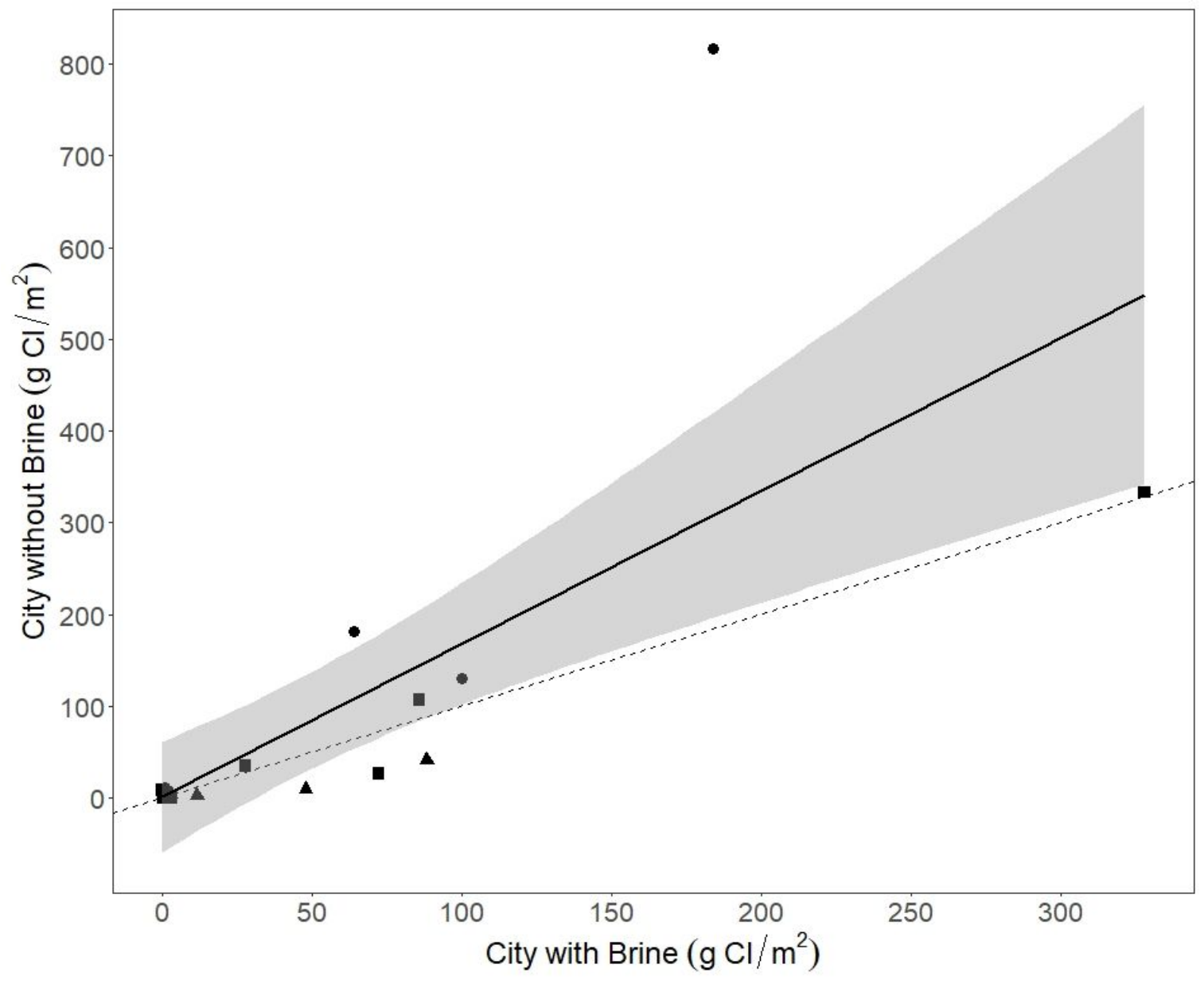


Figure S3. Linear regression of chloride loads for each winter in paired cities. Each point represents the chloride load from a pair of cities during one winter season (triangles:

Ferguson/Jennings; squares: Manchester/Ballwin; circles: Rock Hill/Webster Groves). The dashed 1:1 line represents equal salt application in paired cities. The regression line and $95 \%$ confidence interval DO NOT take into account the circled data points from the Ferguson/Jennings pair shown as triangles.

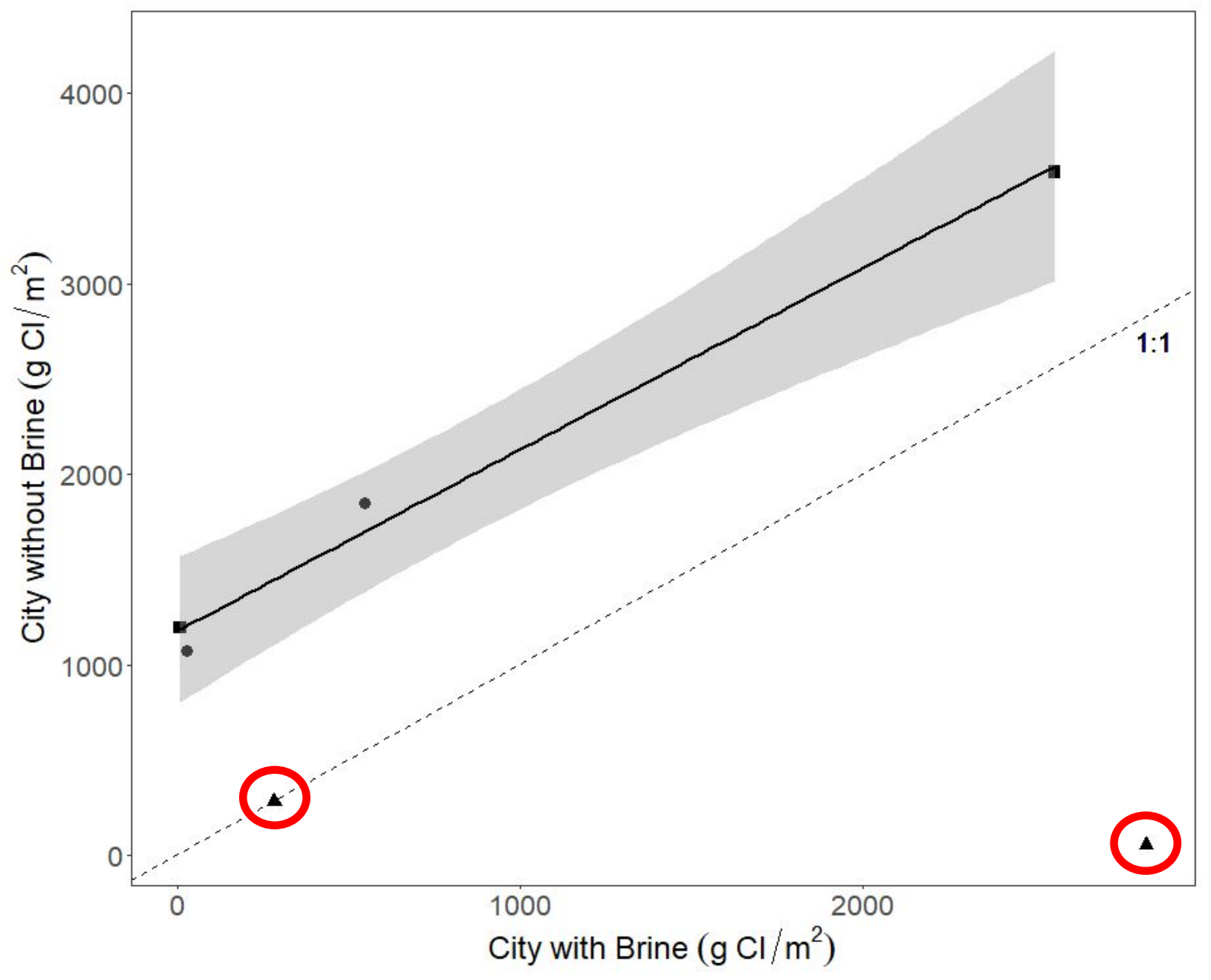


Table S1. Municipal characteristics from the six study cities as well as three additional randomly selected cities of similar land area in St. Louis County.

\begin{tabular}{|c|c|c|c|c|c|c|c|c|c|c|c|c|c|c|c|c|}
\hline & 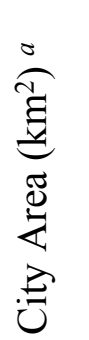 & 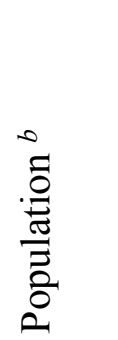 & 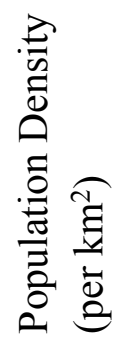 & 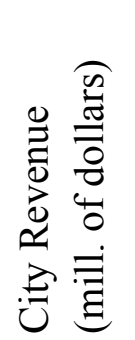 & 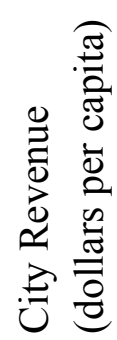 & 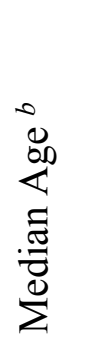 & $\frac{0}{3} \underbrace{0}_{0}$ & 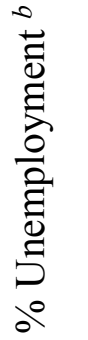 & 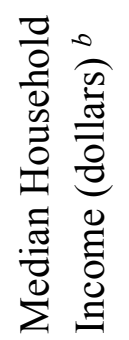 & 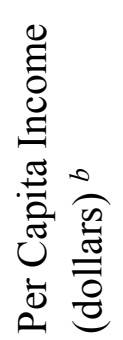 & 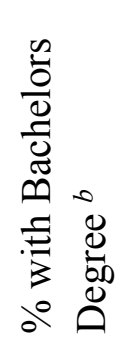 & 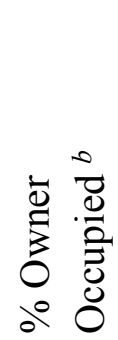 & 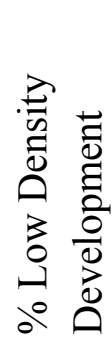 & \begin{tabular}{l}
$\tau$ \\
\multirow{0}{0}{} \\
0 \\
0 \\
0 \\
0 \\
0
\end{tabular} & $\begin{array}{l}\Sigma \\
\text { ] } \\
0 \\
0 \\
0 \\
0 \\
0\end{array}$ & 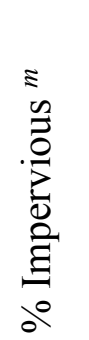 \\
\hline $\mathrm{M}$ & 13.1 & 18183 & 1390 & $20.14^{c}$ & 1107 & 37.9 & 83.1 & 3.0 & 77978 & 38734 & 54.6 & 77.7 & 76.1 & 6.0 & 9.1 & 32.4 \\
\hline B & 23.2 & 30431 & 1314 & $22.67 d$ & 745 & 42.2 & 88.0 & 2.9 & 86484 & 41885 & 54.7 & 80.4 & 80.8 & 6.7 & 7.9 & 29.7 \\
\hline $\mathrm{F}$ & 16.0 & 21035 & 1318 & $17.92^{e}$ & 852 & 35.9 & 30.4 & 14.4 & 31981 & 16662 & 20.2 & 56.9 & 83.1 & 0.0 & 7.6 & 30.9 \\
\hline $\mathrm{J}$ & 9.7 & 14749 & 1521 & $10.48^{f}$ & 710 & 33.3 & 5.8 & 21.4 & 31981 & 16662 & 10.0 & 49.9 & 78.8 & 0.1 & 8.9 & 31.8 \\
\hline $\mathrm{R}$ & 2.8 & 4628 & 1630 & $5.47^{g}$ & 1182 & 38.2 & 76.5 & 2.2 & 64306 & 36425 & 55.5 & 90.7 & 81.8 & 0.0 & 9.4 & 40.6 \\
\hline $\mathrm{W}$ & 15.3 & 23111 & 1511 & $22.43^{h}$ & 971 & 39.9 & 90.6 & 2.6 & 85856 & 44263 & 65.1 & 80.1 & 89.8 & 0.0 & 9.5 & 34.2 \\
\hline $\mathrm{V}$ & 11.0 & 6942 & 632 & $7.75^{i}$ & 1117 & 34.9 & 85.1 & 2.3 & 54854 & 32595 & 40.1 & 61.0 & 34.6 & 27.1 & 6.6 & 23.5 \\
\hline $\mathrm{N}$ & 11.3 & 10785 & 956 & $6.03^{j}$ & 559 & 37.6 & 20.8 & 7.1 & 38472 & 19696 & 16.1 & 64.0 & 70.6 & 14.1 & 7.4 & 23.9 \\
\hline $\mathrm{U}$ & 15.3 & 35371 & 2316 & $29.97^{k}$ & 847 & 37.4 & 50.8 & 4.8 & 56281 & 45517 & 55.6 & 52.2 & 81.9 & 0.0 & 6.4 & 34.8 \\
\hline
\end{tabular}

$\mathrm{M}=$ Manchester, $\mathrm{B}=$ Ballwin, $\mathrm{F}=$ Ferguson, $\mathrm{J}=$ Jennings, $\mathrm{R}=$ Rock Hill, $\mathrm{W}=$ Webster Groves, $\mathrm{V}=$ Valley Park, N=Bellefontaine Neighbors, $\mathrm{U}=$ University City

Data sources:

a St. Louis County Government http://data.stlouisco.com/datasets/2273a9e78bae4b94a2a1e6bf67e375e1_6

${ }^{b}$ US Census Bureau, 2016 American Community Survey

c 2017 Comprehensive Annual Financial Report, Manchester, Mo. https://www.manchestermo.gov/ArchiveCenter/ViewFile/Item/69

${ }^{d}$ City of Ballwin, Missouri, Comprehensive Annual Financial Report For the Year Ended December 31, 2017.

https://www.ballwin.mo.us/pageimages/DocumentCenter/Financials/Financial_Statement_17.pdf 
e City of Ferguson, Missouri, Comprehensive Annual Financial Report For the Year Ended June 30, 2017. http://www.fergusoncity.com/DocumentCenter/View/2990/FY2017-City-Ferguson-CAFR?bidId=

$f$ City of Jennings Financial Report (Audited), Year Ended March 31, 2016. http://www.cityofjennings.org/vimages/shared/vnews/stories/58e7fc3865cd6/2105-2016\%20Financial\%20Stmt.pdf

$g$ Comprehensive Annual Financial Report For Fiscal Year Ended March 31, 2015, City of Rock Hill, Missouri. http://www.rockhillmo.net/uploads/Departments/RH\%20FY15\%20CAFR\%20Final.pdf

${ }^{h}$ City of Webster Groves, Missouri, Comprehensive Annual Financial Report For the Year Ended June 30, 2017. https://webstergroves.org/DocumentCenter/View/6510/2017-Comprehensive-Annual-Financial-Report?bidId=

${ }^{i}$ City of Valley Park, Missouri, Audit of Financial Statements, Year Ended June 30, 2017. http://www.valleyparkmo.org/wpcontent/uploads/2018/02/FY-2017-Audit.pdf

${ }^{j}$ City of Bellefontaine Neighbors, Missouri, Financial Report (Audited), Year Ended June 30, 2017. https://www.cityofbn.com/files/8515/1128/8166/Year_Ending_June_30_2017.pdf

${ }^{k}$ City of University City, Missouri, Comprehensive Annual Financial Report For the Fiscal Year Ended June $30,2017$. https://www.ucitymo.org/DocumentCenter/View/12482/City-of-University-City---CAFR-2017---FINAL?bidId=

${ }^{l}$ National Land Cover Database (NLCD 2011)

${ }^{m}$ Metropolitan St. Louis Sewer District proprietary shapefile coverage 
Table S2. Standardized metrics from Table S1. Standardized values were calculated as z-scores which results in a column mean of 0 and standard deviation of 1 for each metric.

\begin{tabular}{|c|c|c|c|c|c|c|c|c|c|c|c|c|c|}
\hline & 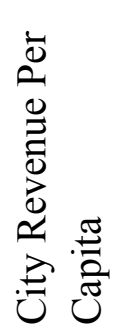 & 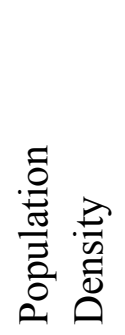 & 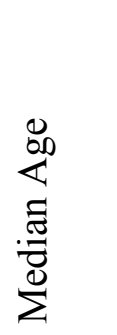 & $\frac{. \stackrel{0}{Z}}{3}$ & 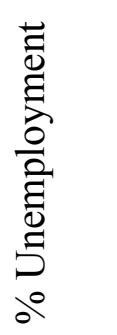 & 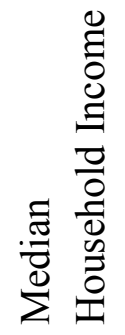 & 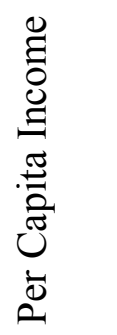 & 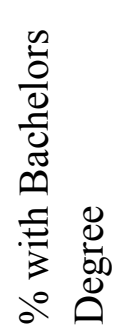 & 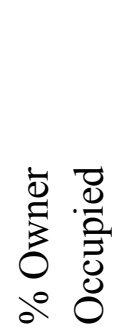 & 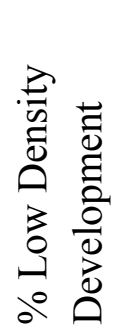 & 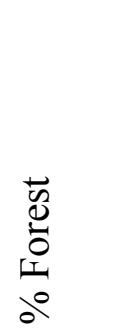 & 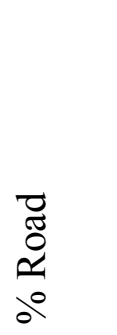 & $\begin{array}{l}0 \\
.0 \\
0 \\
0 \\
0 \\
\Xi \\
\vdots \\
0\end{array}$ \\
\hline $\mathrm{M}$ & 0.99 & -0.02 & 0.16 & 0.74 & -0.56 & 0.89 & 0.53 & 0.64 & 0.66 & 0.05 & 0.00 & 0.86 & 0.20 \\
\hline $\mathrm{B}$ & -0.73 & -0.18 & 1.79 & 0.89 & -0.57 & 1.28 & 0.80 & 0.65 & 0.85 & 0.34 & 0.08 & -0.13 & -0.31 \\
\hline $\mathrm{F}$ & -0.22 & -0.17 & -0.60 & -0.87 & 1.14 & -1.23 & -1.34 & -1.03 & -0.77 & 0.49 & -0.65 & -0.45 & -0.07 \\
\hline $\mathrm{J}$ & -0.89 & 0.26 & -1.59 & -1.62 & 2.17 & -1.23 & -1.34 & -1.52 & -1.26 & 0.22 & -0.64 & 0.69 & 0.08 \\
\hline $\mathrm{R}$ & 1.34 & 0.50 & 0.27 & 0.53 & -0.67 & 0.26 & 0.33 & 0.69 & 1.56 & 0.40 & -0.65 & 1.12 & 1.74 \\
\hline $\mathrm{W}$ & 0.34 & 0.24 & 0.92 & 0.96 & -0.61 & 1.25 & 1.00 & 1.16 & 0.83 & 0.90 & -0.65 & 1.18 & 0.55 \\
\hline $\mathrm{V}$ & 1.03 & -1.65 & -0.98 & 0.80 & -0.66 & -0.18 & 0.01 & -0.06 & -0.49 & -2.52 & 2.27 & -1.26 & -1.46 \\
\hline $\mathrm{N}$ & -1.61 & -0.95 & 0.05 & -1.17 & 0.05 & -0.93 & -1.08 & -1.23 & -0.28 & -0.29 & 0.87 & -0.61 & -1.40 \\
\hline $\mathrm{U}$ & -0.25 & 1.98 & -0.03 & -0.25 & -0.29 & -0.11 & 1.10 & 0.69 & -1.10 & 0.41 & -0.65 & -1.39 & 0.65 \\
\hline
\end{tabular}

$\mathrm{M}=$ Manchester, $\mathrm{B}=$ Ballwin, $\mathrm{F}=$ Ferguson, $\mathrm{J}=$ Jennings, $\mathrm{R}=$ Rock Hill, $\mathrm{W}=$ Webster Groves, $\mathrm{V}=$ Valley Park, N=Bellefontaine Neighbors, $\mathrm{U}=$ University City 
Table S3. Characteristics of each pipeshed and sampling location. The asterisk $(*)$ denotes a site that was removed from the study.

\begin{tabular}{|c|c|c|c|c|c|}
\hline Site ID & Drainage Area (ha) & Impervious Area (\%) & Road Area (\%) & Pipe Diameter $(\mathrm{cm})$ & Pipe Slope $(\mathrm{cm} / \mathrm{cm})$ \\
\hline M1 & 7.5 & 30.6 & 10.1 & 60.96 & 0.0111 \\
\hline M2 & 14.6 & 23.3 & 6.9 & 91.44 & 0.0167 \\
\hline M3 & 5.3 & 36.7 & 11.9 & 83.82 & 0.0167 \\
\hline M4 & 5.2 & 26.6 & 6.8 & 91.44 & 0.0361 \\
\hline $\mathrm{B} 1$ & 9.9 & 38.2 & 10.5 & 83.82 & 0.0444 \\
\hline B2 & 13.0 & 37.2 & 8.0 & 106.68 & 0.0167 \\
\hline B3 & 5.2 & 35.4 & 13.0 & 60.96 & 0.0083 \\
\hline B4 & 5.6 & 32.0 & 8.9 & 76.2 & 0.0333 \\
\hline F1 & 10.0 & 29.9 & 10.6 & 76.2 & 0.0861 \\
\hline F2 & 7.0 & 31.0 & 11.3 & 68.58 & 0.0361 \\
\hline F3 & 12.5 & 35.5 & 13.8 & 119.38 & 0.0028 \\
\hline F4 & 12.0 & 34.7 & 7.9 & 63.5 & 0.0639 \\
\hline $\mathrm{J} 1$ & 13.3 & 28.9 & 12.3 & 106.68 & 0.0306 \\
\hline $\mathrm{J} 2$ & 16.7 & 31.0 & 11.1 & 91.44 & 0.0278 \\
\hline $\mathrm{J} 3$ & 13.2 & 30.9 & 3.9 & 76.2 & 0.0250 \\
\hline $\mathrm{J} 4$ & 1.8 & 37.6 & 11.9 & 53.34 & 0.0361 \\
\hline $\mathrm{R} 1$ & 6.0 & 37.1 & 11.2 & 91.44 & 0.0722 \\
\hline $\mathrm{R} 2$ & 17.1 & 30.8 & 7.7 & 91.44 & 0.0417 \\
\hline R3 & 5.9 & 36.3 & 10.0 & 68.58 & 0.0167 \\
\hline $\mathrm{R} 4$ & 3.6 & 36.8 & 12.1 & 45.72 & 0.0167 \\
\hline W1 & 13.9 & 33.0 & 10.3 & 91.44 & 0.0639 \\
\hline W2 & 8.9 & 32.9 & 12.6 & 73.66 & 0.0389 \\
\hline W3 & 11.7 & 33.4 & 11.0 & 91.44 & 0.0222 \\
\hline $\mathrm{W} 4 *$ & 0.1 & 44.7 & 27.3 & 30.48 & 0.0028 \\
\hline W5 & 11.7 & 31.4 & 9.5 & 68.58 & 0.0069 \\
\hline
\end{tabular}


Table S4. NOAA Climate Data Online (https://www.ncdc.noaa.gov/cdo-web/) weather stations used in this study.

\begin{tabular}{|l|l|l|l|l|}
\hline Station ID & Station Name & Latitude & Longitude & Elevation (m) \\
\hline USC00237398 & St Charles 7 SSW, MO US & 38.6856 & -90.5231 & 137.2 \\
\hline USC00237452 & St Louis Science Center, MO US & 38.6291 & -90.2706 & 166.1 \\
\hline USW00003966 & St Louis Spirit of St Louis Airport, MO US & 38.65722 & -90.65583 & 140.8 \\
\hline USW00013994 & St Louis Lambert International Airport, MO US & 38.7525 & -90.3736 & 161.8 \\
\hline US1MOFSA189 & Edmunson 0.7 NNW, MO US & 38.7425 & -90.3721 & 177.1 \\
\hline US1MOSL0020 & Ladue 1.6 N, MO US & 38.6601 & -90.3816 & 189.6 \\
\hline US1MOSL0027 & Ballwin 0.5 NNW, MO US & 38.6008 & -90.5548 & 199 \\
\hline US1MOSL0049 & Ballwin 1.6 E, MO US & 38.5914 & -90.5235 & 173.4 \\
\hline US1MOSL0050 & Webster Groves 1.6 NNE, MO US & 38.6066 & -90.3406 & 153.3 \\
\hline US1MOSL0054 & Webster Groves 0.4 N, MO US & 38.5918 & -90.3531 & 161.2 \\
\hline
\end{tabular}


Table S5. Eigenvectors of the first two axes of the principal component analysis (PCA). Items in bold are considered the most influential for that axis. PC1 accounts for $48.8 \%$ of the variance; PC2 accounts for $27.6 \%$ of the variance.

\begin{tabular}{|l|l|l|}
\hline & PC1 & PC2 \\
\hline Revenue per capita & 0.2298 & 0.1273 \\
\hline Population per km ${ }^{2}$ & 0.1250 & $\mathbf{- 0 . 4 0 0 7}$ \\
\hline Median age & $\mathbf{0 . 3 0 6 1}$ & 0.0050 \\
\hline \% white & $\mathbf{0 . 3 4 6 7}$ & 0.2327 \\
\hline \% unemployed & $\mathbf{- 0 . 3 1 3 8}$ & -0.2717 \\
\hline Median household income & $\mathbf{0 . 3 7 0 3}$ & 0.0865 \\
\hline Per capita income & $\mathbf{0 . 3 5 4 5}$ & 0.0618 \\
\hline \% with bachelor's degree & $\mathbf{0 . 3 8 2 7}$ & 0.0462 \\
\hline \% owner occupied housing & $\mathbf{0 . 3 2 7 9}$ & 0.0355 \\
\hline \% low-density development & 0.1226 & $\mathbf{- 0 . 4 6 9 0}$ \\
\hline \% forest & -0.0914 & $\mathbf{0 . 5 0 9 0}$ \\
\hline \% road & 0.1712 & -0.2466 \\
\hline \% impervious & 0.2230 & $\mathbf{- 0 . 3 7 5 1}$ \\
\hline
\end{tabular}

